\title{
10
}

\section{Neutron star crusts with magnetic fields}

\author{
D.G. YAKOVLEV
}

Ioffe Institute of Physics and Technology, 194021 St. Petersburg, Russia

A.D. KAMINKER

Ioffe Institute of Physics and Technology, 194021 St. Petersburg, Russia

\begin{abstract}
The properties of plasma in neutron star crusts with strong magnetic fields $B=10^{10}-10^{13} \mathrm{G}$ are reviewed: thermodynamic properties (equation of state, entropy, specific heat), transport properties (electron thermal and electrical conductivity of degenerate electron gas, radiative thermal conductivity of very surface nondegenerate layers) and neutrino energy losses. Classical effects of electron Larmor rotation in a magnetic field are considered as well as quantum effects of the electron motion (Landau levels). The influence of the magnetic fields on density and temperature profiles in the surface layers of neutron stars and on neutron star cooling is briefly discussed.
\end{abstract}

Nous présentons la revue des proprietés du plasma dans l'écorce des étoiles neutroniques avec des champs magnétiques forts $B=10^{10}-10^{13} \mathrm{G}$ : proprietés thermodynamiques (equation d'état, entropie, chaleur specifique), proprietés de transfer (conductivité electronique thermique et electrique du gaz electronique dégénéré, conductivité radiative thermique des couches non-dégénées superficielles), et les pertes dûes à l'énergie des neutrinos. Nous examinons des effets classiques de la rotation Larmor d'un electron dans le champ magnétique, et aussi des effets quantiques (niveaux de Landau ). Nous discutons en bref l'influence des champs magnétiques sur la densité et la temperáture des couches des étoiles neutroniques et sur les taux de refroidissement des étoiles neutriniques. 


\subsection{Introduction}

Neutron stars are the densest stars known in the Universe. Their masses are $M \sim 1.4 M_{\odot}$, and radii $R \sim 10 \mathrm{~km}$. The mass density of matter $\rho$ in neutron star cores is several times larger than the standard nuclear density, $\rho_{0}=2.8 \times 10^{14} \mathrm{~g} \mathrm{~cm}^{-3}$. The properties of this superdense matter (equation of state, nuclear composition, etc.; see Shapiro and Teukolsky 1983) are known poorly and attract attention of many scientists. These properties cannot be reproduced in laboratory but can be studied by astrophysical methods comparing theoretical models of processes in neutron stars (e.g., cooling of an isolated star) with observational data.

Neutron star cores which contain superdense matter are surrounded by envelopes (crusts) of lower density, $\rho<\rho_{0}$. The crusts play a key role in many processes which are observed and studied theoretically. Many neutron stars possess strong magnetic fields, $B=10^{10}-10^{13} \mathrm{G}$. The aim of this work is to review briefly the effects of the magnetic fields in neutron star crusts. The subject is studied since the first works of Canuto and co-authors in 70-s (Canuto and Ventura 1977).

\subsection{Neutron Star Crusts without Magnetic Fields}

Let us start with a brief description of neutron star crusts without magnetic fields. The crust extends from the the bottom of the atmosphere to the dense core; its thickness is several $\mathrm{km}$ (Shapiro and Teukolsky 1983). It can be divided into the outer crust, $\rho<\rho_{d}$, and the inner crust $\rho>\rho_{d}$, where $\rho_{d} \approx 4 \times 10^{11} \mathrm{~g} \mathrm{~cm}^{-3}$ is the neutron drip density. Matter of the outer crust consists of electrons and atoms. The electrons can be nondegenerate in a very thin surface layer, and they are strongly degenerate deeper in the star. The atoms are fully ionized by the electron pressure (they are actually bare nuclei) everywhere except near the very surface.

In the inner crust, free neutrons appear (in addition to the electrons and nuclei) owing to the drip from the nuclei. The neutrons are degenerate and superfluid. The superfluidity is caused by the Cooper pairing of the neutrons due to nuclear forces; corresponding critical temperature is about $10^{8}-10^{10} \mathrm{~K}$ (e.g., Wambach et al., 1991). The rotation of the superfluid component of matter in rotating neutron stars is realized in the form of quantized vortices which are parallel to the rotational axis. The interaction of the vortices and the nuclei (pinning and depinning) in the inner crust is thought to be responsible for pulsar glitches (e.g., Pines 1991). The properties of nuclei and free neutrons are model dependent (Baym et al 1971, Negele and Vautherin 1973) and rather uncertain. At the bottom 


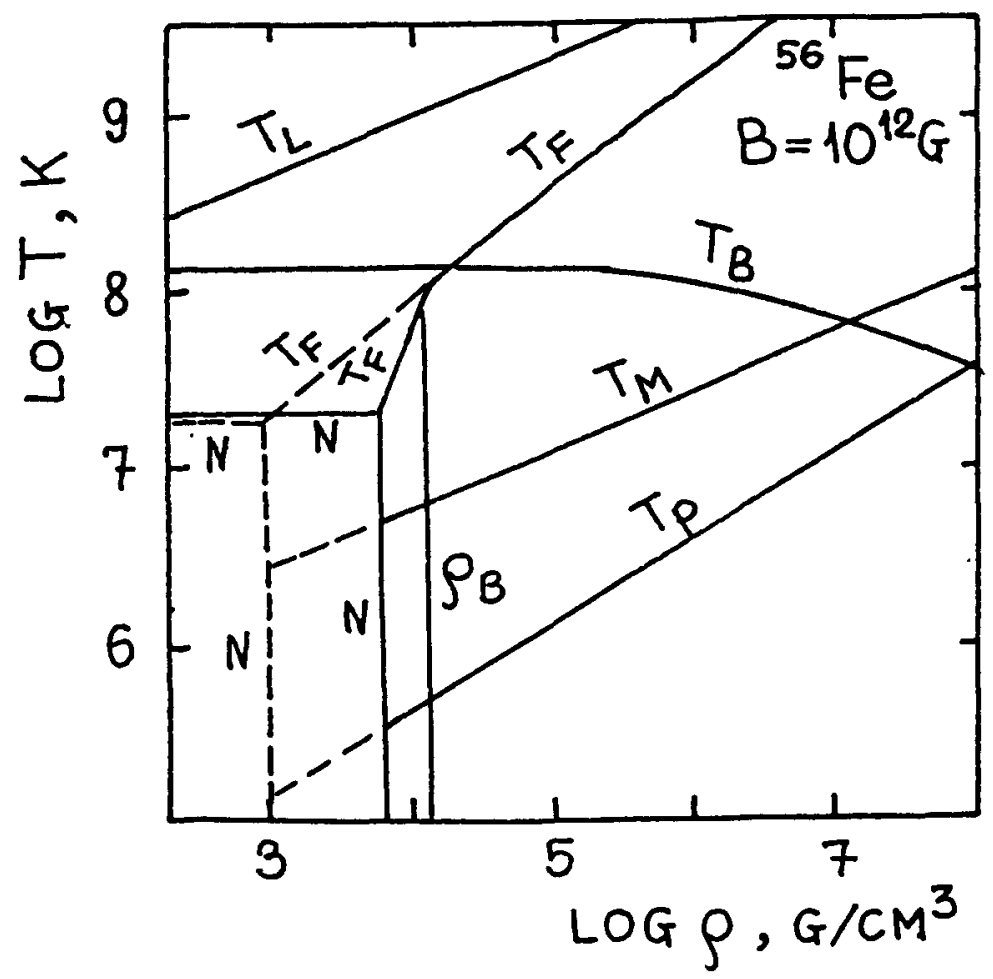

Fig. $10.1 \rho-T$ diagram of Fe matter for $B=10^{12} \mathrm{G}$. $T_{F}$ is the electron degeneracy temperature, $T_{l}$ corresponds to $\Gamma=1, T_{m}$ is the melting temperature, $T_{p}$ is the ion plasma temperature; $T_{B}$ is given by $(8) ; \rho_{B}$ is explained in Sec. 3. Lines $N$ restrict the low- $T$ low- $\rho$ domain of incomplete ionization and electron gas non-ideality. Dashes show the curves for $B=0$

of the inner crust, the nuclei may form clusters and droplets with strongly nonspherical shapes (Lorenz et al. 1993). Below we shall mainly consider the outer crusts.

The state of electrons is determined by the electron Fermi momentum $p_{F 0}$ and 'relativistic parameter' $x$ :

$$
p_{F 0}=\hbar\left(3 \pi^{2} n_{e}\right)^{1 / 3}, x=\frac{p_{F 0}}{m_{e} c} \approx 1.009\left(\frac{\rho_{6}}{\mu_{e}}\right)^{1 / 3},
$$

where $n_{e}$ is the electron number density, $\rho_{6}$ is density in units of $10^{6} \mathrm{~g} \mathrm{~cm}^{-3}$, and $\mu_{e}$ is the number of nucleons per one electron. For $x \ll 1\left(\rho_{6} \ll 1\right)$ the electron gas is non-relativistic, while for $x \gg 1$ it is relativistic. The electron degeneracy temperature $T_{F}$ is (Fig. 1) $T_{F}=T_{0}\left(\sqrt{1+x^{2}}-1\right.$ ), where $T_{0}=m_{e} c^{2} / k_{B} \approx 5.930 \times 10^{9} \mathrm{~K}, k_{B}$ is the Boltzmann constant, and $m_{e}$ the electron mass. 
The state of the ions is characterized by the ion-coupling parameter

$$
\Gamma=\frac{Z^{2} e^{2}}{a k_{B} T} \approx 0.2275 \frac{Z^{5 / 3}}{T_{8}}\left(\frac{\rho_{6}}{\mu_{e}}\right)^{1 / 3}
$$

where $a=\left[3 /\left(4 \pi n_{i}\right)\right]^{1 / 3}$ is the radius of ion sphere (the charge of electrons within the sphere compensates the ion charge), $n_{i}$ is the ion number density, and $T_{8}=T /\left(10^{8} \mathrm{~K}\right)$. For simplicity, we consider one component plasma of ions. Some properties of multi-component ion mixtures in neutron star crusts are reviewed by Yakovlev and Shalybkov (1989).

At sufficiently high temperatures the ions form a classical Boltzmann gas. With decreasing $T$, the gas gradually (without any phase transition) becomes a Coulomb liquid, and then (with a phase transition) a Coulomb crystal. The gaseous regime occurs (Hansen 1973) for $\Gamma \ll 1\left(T \gg T_{l}\right.$, Fig. 1). The classical Coulomb crystal melts (Nagara et al. 1987) at $\Gamma \approx 172$ $\left(T=T_{m}\right)$. The thermodynamics of strongly coupled Coulomb systems has been studied extensively by Monte Carlo and other methods (see Hansen 1973, Pollock and Hansen 1973, Hansen et al. 1977, Slattery et al. 1980, 1982 , and references therein).

At low $T$ the quantum effects in ion motion (zero-point ion vibrations) become important. These effects are especially pronounced if $T \ll T_{p}$, where

$$
T_{p}=\frac{\hbar \omega_{p}}{k_{B}} \approx 7.832 \times 10^{6}\left(\frac{\rho_{6}}{\mu_{e}^{2}}\right)^{1 / 2} \mathrm{~K}, \omega_{p}^{2}=\frac{4 \pi Z^{2} e^{2} n_{i}}{m_{i}}
$$

$\omega_{p}$ is the ion plasma frequency, and $m_{i}$ is the ion mass. The amplitude of zero-point vibrations is commonly much smaller than the typical interion distance, $a$. With increasing density, the amplitude to $a$ ratio becomes larger, and at large $\rho$ zero-point vibrations can prevent crystallization (Mochkovitch and Hansen 1979, Ceperley and Alder 1980). This effect is especially important for $\mathrm{H}$ and He. Note that the Debye temperature of the classical Coulomb crystal is $\approx 0.45 T_{p}$ (Carr 1961).

For small $\rho$ and $T$, the effects of incomplete ionization and electron gas non-ideality become important. This domain is restricted schematically by lines $N$ in Fig. 1. The lines correspond to $\max \left(T_{F}, T\right)=\left|\epsilon_{e}\right|$, where $T_{F}$ is calculated in the free electron gas approximation and $\epsilon_{e}$ is the mean energy per electron for isolated atoms in the Thomas-Fermi approximation. 


\subsection{Magnetic Field Effects}

The properties of matter in neutron star crusts can be affected by the magnetic fields. The magnetic field effects are numerous. Below we consider two major effects: the classical electron Larmor rotation, and the Landau quantization of electron motion.

The classical effects are described by the Hall magnetization parameter,

$$
\eta_{0}=\omega_{B}^{*} \tau_{0} \approx 1.76 \times 10^{3} \frac{m_{e}}{m_{*}} B_{12}\left(\frac{\tau_{0}}{10^{-16_{s}}}\right), \omega_{B}^{*}=\frac{|e| B}{m_{*} c},
$$

where $\tau_{0}$ is the effective electron relaxation time (Sec. 5.2), $\omega_{B}^{*}$ is the electron gyrofrequency, $B_{12}=B /\left(10^{12} \mathrm{G}\right)$, and $m_{*}$ is the effective electron mass $\left(m_{*}=m_{e}\right.$ for nonrelativistic electron gas; $m_{*}=\sqrt{m_{e}^{2}+\left(p_{F} / c\right)^{2}}$ for strongly degenerate gas). The classical effects are strong when $\eta_{0} \gg 1$ (electrons suffer many Larmor rotations between successive collisions). The $\rho-T$ domain where the electrons are magnetized $\left(\eta_{0} \gg 1\right)$ is commonly wide. For instance the field $B=10^{12} \mathrm{G}$ magnetizes the electrons almost for all $\rho$ and $T$ shown in Fig. 1.

The quantum effects are associated with quantization of electron motion transverse to the magnetic field. If the Landau gauge of the magnetic field vector potential $(A=(-B y, 0,0))$ is used, an electron state can be characterized (Klepikov 1954, Kaminker and Yakovlev 1981) by four quantum numbers, $p_{z}, n, s$ and $p_{x}$. In this case $p_{z}$ is an electron momentum along $\mathbf{B}$, $n=0,1, \ldots$ enumerates the Landau levels, $s$ is the sign of the projection of the electron spin onto the momentum $\left(s= \pm 1\right.$ for $n>0 ; s=-\operatorname{sign}\left(p_{z}\right)$ for $n=0)$ and $p_{x}$ determines an $y$-coordinate of the electron Larmor guiding center. Then the electron energy is

$$
\varepsilon=\sqrt{m_{e}^{2} c^{4}+c^{2} p_{z}^{2}+2 n m_{e} c^{2} \hbar \omega_{B}}, \hbar \omega_{B}=m_{e} c^{2} b, \quad b=\frac{B}{B_{c}},
$$

where $\omega_{B}=|e| B /\left(m_{e} c\right)$ is the electron cyclotron frequency, and $B_{c}=$ $4.414 \times 10^{13} \mathrm{G}$ is the 'relativistic' magnetic field $\left(\hbar \omega_{B c}=m_{e} c^{2}\right)$.

The number density of the free electron gas is related to the electron chemical potential $\mu$ by the equation

$$
n_{e}=\frac{m_{e} \omega_{B}}{(2 \pi \hbar)^{2}} \sum_{n s} \int_{-\infty}^{+\infty} f \mathrm{~d} p_{z}, f=\left[1+\exp \left(\frac{\varepsilon-\mu}{k_{B} T}\right)\right]^{-1},
$$

where $f$ is the Fermi-Dirac distribution. The electron degeneracy temperature is $T_{F}=T_{0}\left(\sqrt{1+x_{B}^{2}}-1\right)$, where the 'relativistic parameter' $x_{B}=$ $p_{F} /\left(m_{e} c\right)$ depends generally on $B$. Using (6) one can easily show that the strongly degenerate electrons $\left(T \ll T_{F}\right)$ populate the only ground Landau 
level when density is not too high, $\rho<\rho_{B}$, where $\rho_{B}=2.066 \times 10^{6} b^{3 / 2} \mu_{e}$ $\mathrm{g} \mathrm{cm}^{-3}$. In this case

$$
p_{F}=\frac{2 \pi^{2} \hbar^{2} n_{e}}{m_{e} \omega_{B}}, x_{B}=\frac{2 x^{3}}{3 b},
$$

where $x$ is defined by (1). In the limit of $\rho \ll \rho_{B}$ the magnetic field strongly reduces the electron Fermi energy and increases the density domain of incomplete ionization and electron gas non-ideality (Yakovlev 1981a, Hernquist 1984, Van Riper 1988). If $\rho \gg \rho_{B}$, strongly degenerate electrons populate many Landau levels and the Fermi energy is almost independent of $B\left(x_{B} \approx x\right)$.

For further analysis, it is convenient to introduce the temperature

$$
T_{B}=\frac{\hbar \omega_{B}^{*}}{k_{B}} \approx 1.34 \times 10^{8} B_{12} \frac{m_{e}}{m_{*}} \mathrm{~K} .
$$

When $T \gg T_{B}$ the electrons populate many Landau levels for any $\rho$ due to high thermal energy. In this case the thermal widths of the Landau levels $\left(\sim k_{B} T\right)$ are higher than the inter-level spacing and the magnetic field acts as a non-quantizing one regardless of the electron degeneracy.

In the domain of $T<T_{B}$ and $\rho<\rho_{B}$ (separated into subdomains of degenerate and nondegenerate gas) the electrons populate mostly the ground Landau level. In this domain the magnetic field acts as a strongly quantizing one and modifies essentially all the properties of matter.

Finally, in the domain of $T<T_{B}$ and $\rho \gg \rho_{B}$ the electrons are degenerate and populate many Landau levels but the inter-level spacing exceeds $k_{B} T$. Then the magnetic field is slightly quantizing. It does not affect the bulk properties (pressure, chemical potential) which are determined by all the Fermi sea of electrons but affects quantities determined by thermal electrons near the Fermi level (heat capacity, entropy, transport properties).

We will assume below that the magnetic fields do not affect motions of ions, for instance, the phonon spectrum of the crystal. This is so (e.g., Usov et al. 1980) if $\omega_{B i}=Z e B /\left(m_{i} c\right) \ll \omega_{p}$, i.e. if $B \ll 10^{14} \sqrt{\rho_{6}} \mathrm{G}$.

\subsection{Thermodynamics of Matter in High Magnetic Fields} Thermodynamic properties of plasma are affected by the magnetic fields if the fields are quantizing. We will discuss the case of strong degeneracy $\left(T \ll T_{F}\right)$.

The basic approach consists in obtaining the grand potential $\Omega=-P V$ 


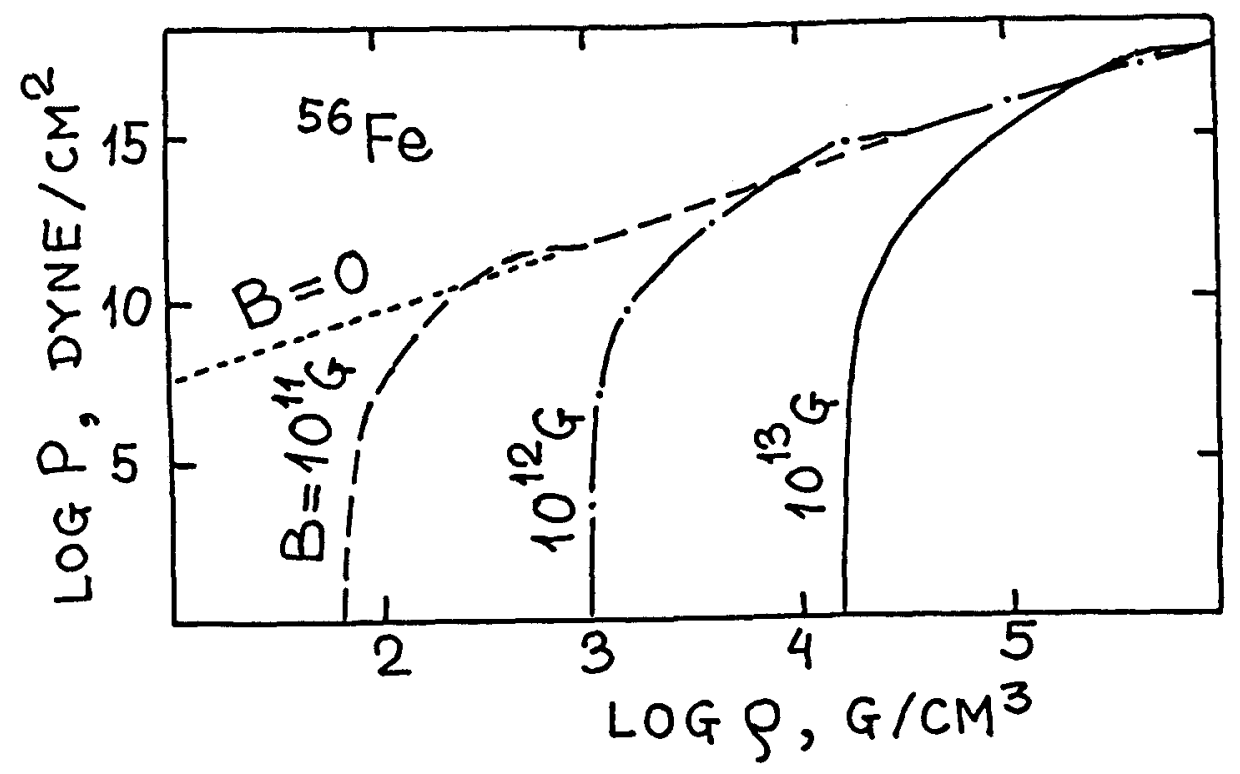

Fig. 10.2 Equation of state of matter at $T=0$ (Rögnvaldsson et al. 1993)

( $V$ is normalization volume) which generates other thermodynamic quantities (Landau and Lifshitz 1980). In the free electron gas approximation

$$
P=-\frac{m_{e} \omega_{B} k_{B} T}{(2 \pi \hbar)^{2}} \sum_{n s} \int_{-\infty}^{+\infty} \ln (1-f) \mathrm{d} p_{z},
$$

which yields Eq. (6) since $n_{e}=\partial P / \partial \mu$.

With increasing $\rho$ (and/or decreasing $B$ ), the thermodynamic quantities show features or oscillations of van Alphen - de Haas type. The features occur when degenerate electrons populate new excited Landau levels $n$ (because the electron density of states $\mathrm{d} p_{z} / \mathrm{d} \varepsilon$ possesses square root singularities at $p_{z}=0$ for these $n$, see (5)).

The bulk thermodynamic quantities (pressure, chemical potential) are determined by all electrons $(\varepsilon \leq \mu)$. Accordingly these quantities are greatly affected by strongly quantizing fields but slightly affected by weakly quantizing fields.

The quantities determined by the electrons with energies $|\varepsilon-\mu|<k_{B} T$ show large oscillations under the action of strongly and weakly quantizing fields. These quantities are usually expressed as derivatives of the bulk quantities with respect to thermodynamic variables. The higher the derivative, the larger the oscillations. The examples of strongly oscillating quantities are: specific entropy and heat, magnetization, magnetic susceptibility, elec- 


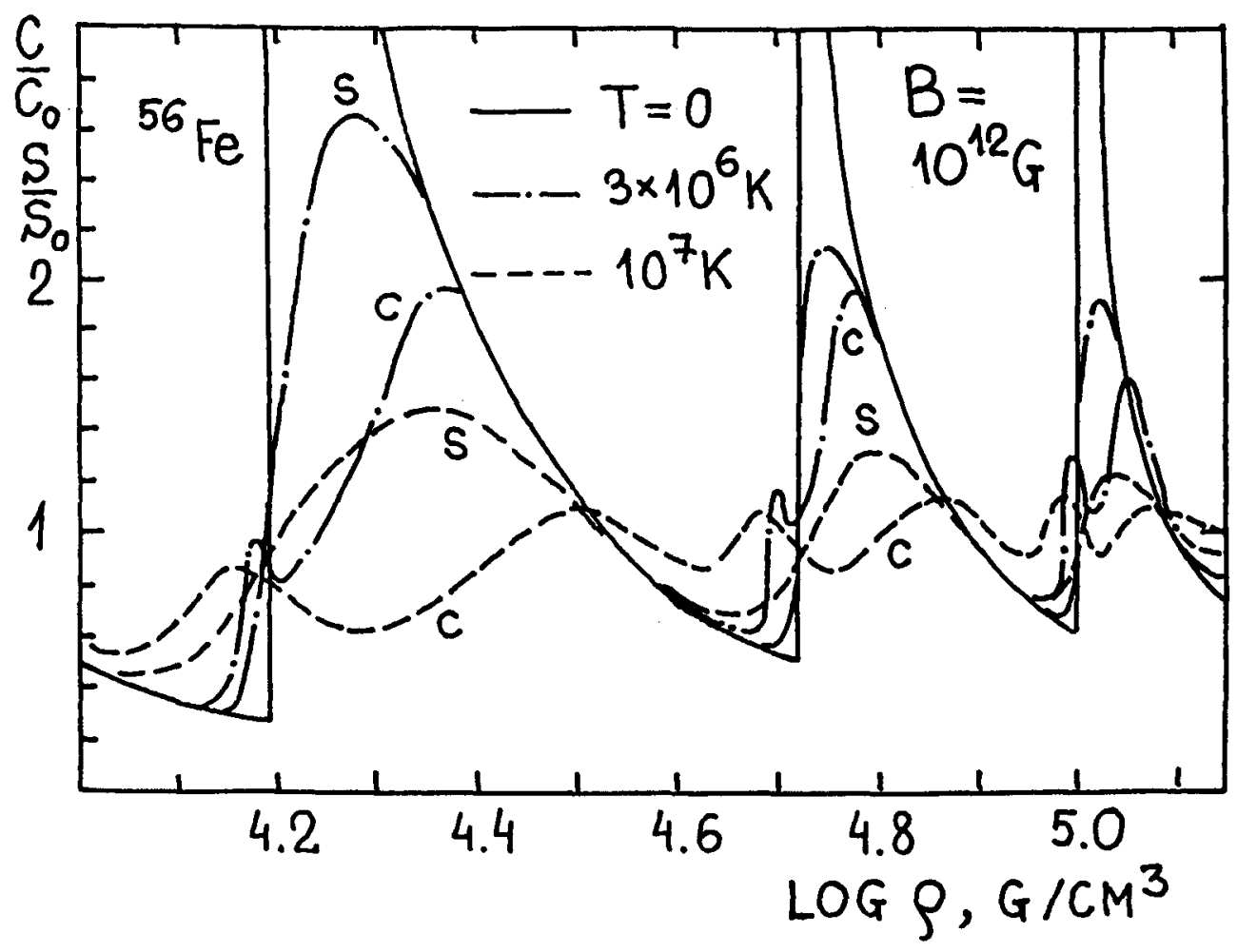

Fig. 10.3 Ratios of electron specific entropy $S(B) / S(0)$ and specific beat $C(B) / C(0)$ versus $\rho$ at $B=10^{12} \mathrm{G}$ for $T \rightarrow 0, T=3 \times 10^{6} \mathrm{~K}$ and $10^{7} \mathrm{~K}$ in the free electron gas model. If $T \rightarrow 0$, one has $S=C$ and formally (in the adopted model) the curves show infinite jumps at those $\rho$ at which new Landau levels are populated. The jumps displayed corresond to population of the levels $n=1,2$ and 3

tron screening length of an electric charge in a plasma (see Yakovlev 1980a, 1984; Blandford and Hernquist 1982 and the references therein).

With increasing $T$, the oscillations become weaker which can be treated as the thermal broadening of the Landau levels. When $T \gg T_{B}$ (and the field becomes nonquantizing) the oscillations are entirely smeared out, and the field-free results are reproduced.

The free-electron approximation (9) can be inaccurate for low $T$ at $\rho \ll$ $\rho_{B}$ and near the peaks of strong oscillations. In these cases one needs more advanced approaches. Recently the bulk properties of cold $(T=0)$ iron matter for $B=10^{10}-10^{13} \mathrm{G}$ have been studied extensively by Rögnvaldsson et al. (1993) using the Thomas-Fermi (TF) approach and taking into account population of several Landau levels. (Also see Rögnvaldsson et al. 1993 for references to earlier works done in the frame of TF and related theo- 
ries.) Another approach to describe strong oscillations at low $T$ is based on introducing some effective widths of the Landau levels caused by electron interactions. This broadening of the Landau levels was studied in the solid state physics (Kubo et al. 1965; Schoenberg 1984) and in the astrophysics (e.g., Yakovlev 1980, Hernquist 1984). In particular, the Landau levels can be broadened by the electron collisions (with width $\gamma \sim \hbar / \tau_{0}$ ). Note that a selfconsistent theory of strong quantum oscillations does not yet exist.

Fig. 2 shows the TF equation of state for cold matter. If $\rho<\rho_{B}$ the electrons populate the ground Landau level and the pressure is essentially lower than for $B=0$ : the magnetic field creates additional binding of electrons. The same effect reduces strongly the electron chemical potential $\mu$. If $B$ is strong, the pressure (in the TF approximation) vanishes at some finite density $\rho_{s}$. For instance, Rögnvaldsson et al. (1993) obtain $\rho_{s}=$ $942 \mathrm{~g} \mathrm{~cm}^{-3}$ for $B=10^{12} \mathrm{G}$. The density $\rho_{s}$ grows with $B$ and can be treated as the surface density of a cold $(T=0)$ neutron star.

Fig. 3 demonstrates quantum oscillations of the electron specific entropy and specific heat. The oscillations of the electron magnetization $M$ (and its derivatives) are even stronger (Blandford and Hernquist 1982). However broadening of the Landau levels prevents the appearance of spontaneous magnetization (the so called Landau orbital ferromagnetism, $B=4 \pi M$ ) under realistic conditions in neutron stars ( $O^{\prime}$ Connel and Roussel 1972, Schmid-Burgk 1973).

\subsection{Transport Properties}

\subsubsection{Electron transport in degenerate electron gas}

Transport properties of the degenerate gas in neutron star crusts are mainly determined by the electrons. The main electron scattering mechanisms are: (i) Coulomb scattering on ions at $T>T_{m}$; (ii) scattering on phonons at $T<T_{m}$; (iii) Coulomb scattering on charged impurities at $T \ll T_{m}$. Below we shall consider cases (i) and (iii), and case (ii) for $T>T_{p}$ (hightemperature phonons). In these cases the scattering is almost elastic: electron energy transfer is negligible in a collision event. For the Coulomb (c) and high- $T$ phonon ( $p h$ ) scatterings, the Fourier transforms $V_{q}$ of the scattering potentials are

$$
\left|V_{q}^{c}\right|^{2}=\left(\frac{4 \pi \delta Z e^{2}}{q^{2}+q_{s}^{2}}\right)^{2},\left|V_{q}^{p h}\right|^{2}=\left\langle r^{2}\right\rangle \frac{\left(4 \pi Z e^{2}\right)^{2}}{3 q^{2}}
$$

where $\hbar / q_{s}$ is the appropriate screening length of electric charge in a plasma (Yakovlev and Urpin 1980, Yakovlev 1980a,b; 1984), $\delta Z=Z$ for case (i), 
$\delta Z=Z_{\text {imp }}-Z$ for case (iii) $\left(Z_{i m p}\right.$ e being the impurity charge), $\left\langle r^{2}\right\rangle=$ $3 k_{B} T u /\left(m_{i} \omega_{p}^{2}\right)$ is the mean squared thermal displacement of an ion in its lattice cite at $T_{p}<T<T_{m}$, and $u \approx 13$ is the appropriate numerical formfactor for a bcc lattice (e.g., Yakovlev and Urpin 1980).

In a magnetic field, all transport coefficients (electric and thermal conductivities $\sigma$ and $\kappa$ as well as the thermopower) are tensors. For instance the thermal conductivity tensor $\hat{\kappa}$ is determined by the longitudinal, transverse, and Hall conductivities $\kappa_{\|}, \kappa_{\perp}$, and $\kappa_{H}$, respectively. Accordingly the thermal flux density is $q=-\kappa_{\|} \nabla_{\|} T-\kappa_{\perp} \nabla_{\perp} T-\kappa_{H} \mathbf{h} \times \nabla T$, where $\nabla_{\|}$and $\nabla_{\perp}$ are the local temperature gradients along and across $\mathbf{B}$, respectively, and $\mathbf{h}=\mathbf{B} / B$. Sometimes the electric resistivity tensor $\hat{\mathcal{R}}=\hat{\sigma}^{-1}$ is more convenient for applications than the electric conductivity tensor $\hat{\sigma}$.

The components of $\hat{\kappa}, \hat{\sigma}$ and $\hat{\mathcal{R}}$ can be expressed as

$$
\begin{aligned}
& \kappa_{\|}=\frac{\pi^{2} k_{B}^{2} T n_{e} \tau_{\|}^{\kappa}}{3 m_{*}}, \quad \kappa_{\perp}=\frac{\pi^{2} k_{B}^{2} T n_{e} \tau_{\perp}^{\kappa}}{3 m_{*}\left(1+\eta_{\kappa}^{2}\right)}, \kappa_{H}=\frac{\pi^{2} k_{B}^{2} T n_{e} \tau_{\perp}^{\kappa} \eta_{\kappa}}{3 m_{*}\left(1+\eta_{\kappa}^{2}\right)}, \\
& \sigma_{\|}=\frac{n_{e} e^{2} \tau_{\|}^{\sigma}}{m_{*}}, \quad \sigma_{\perp}=\frac{e^{2} n_{e} \tau_{\perp}^{\sigma}}{m_{*}\left(1+\eta_{\sigma}^{2}\right)}, \quad \sigma_{H}=\frac{n_{e} e^{2} \tau_{\perp}^{\sigma} \eta_{\sigma}}{m_{*}\left(1+\eta_{\sigma}^{2}\right)}, \\
& \mathcal{R}_{\|}=\frac{m_{*}}{n_{e} e^{2} \tau_{\|}^{\sigma}}, \quad \mathcal{R}_{\perp}=\frac{m_{*}}{n_{e} e^{2} \tau_{\perp}^{\sigma}}, \mathcal{R}_{H}=-\frac{B}{c|e| n_{e}}
\end{aligned}
$$

where $\eta_{\kappa}=\omega_{B}^{*} \tau_{\perp}^{\kappa}$ and $\eta_{\sigma}=\omega_{B}^{*} \tau_{\perp}^{\sigma}$ are the Hall parameters for thermal and electrical conduction, respectively; $\tau_{\|}^{\kappa}$ and $\tau_{\|}^{\sigma}$ are the effective electron relaxation times for the longitudinal transport while $\tau_{\perp}^{\kappa}$ and $\tau_{\perp}^{\sigma}$ are the effective relaxation times for the transverse transport. Thus $\hat{\kappa}, \hat{\sigma}$ and $\hat{\mathcal{R}}$ are determined by four effective relaxation times $\tau_{\|}^{\kappa}, \tau_{\|}^{\sigma}, \tau_{\perp}^{\kappa}, \tau_{\perp}^{\sigma}$ which are generally different, and depend on $T, \rho$, and $B$. The Hall resistivity does not depend on the electron relaxation (under the assumed conditions). This is a nondissipative quantity which describes Hall drift of the magnetic field. The thermopower tensor is defined by two additional parameters (Yakovlev $1980 \mathrm{a}, \mathrm{b} ; 1984)$.

\subsubsection{Nonquantizing magnetic fields}

Let us describe briefly the electron transport in the degenerate electron gas with nonquantizing magnetic fields (Urpin and Yakovlev 1980). In this case all four relaxation times are equal: $\tau_{\|}^{\kappa, \sigma}=\tau_{\perp}^{\kappa, \sigma}=\tau_{0}$, where $\tau_{0}$ is the classical electron relaxation time for $B=0$. Accordingly, the Wiedemann-Franz rule $\hat{\kappa}=\hat{\sigma} \pi^{2} T k_{B}^{2} /\left(3 e^{2}\right)$ is valid for the electron scattering mechanisms under 
discussion. For Coulomb scattering and scattering on high-temperature phonons, one has (Yakovlev and Urpin 1980),

$$
\begin{aligned}
& \tau_{0}^{c}=\frac{p_{F}^{2} v_{F}}{4 \pi(\delta Z)^{2} e^{4} n_{s} L} \approx \frac{5.65 \times 10^{-17}}{x_{i} L \sqrt{1+x^{2}}} \mathrm{~s} \\
& \tau_{0}^{p h}=\frac{\hbar^{2} v_{F}}{e^{2} k_{B} T u}\left(2-\frac{v_{F}^{2}}{c^{2}}\right)^{-1} \approx \frac{8.05 \times 10^{-17} x \sqrt{1+x^{2}}}{\left(2+x^{2}\right) T_{6}} \mathrm{~s},
\end{aligned}
$$

where $x$ is given by (1), $v_{F}$ is the electron Fermi velocity; $n_{s}=n_{i}, x_{i}=Z$ for case (i); $n_{s}=n_{i m p}, x_{i}=(\delta Z)^{2} n_{s} / n_{e}$ for case (iii) ( $n_{i m p}$ being the impurity number density), and $L$ is the Coulomb logarithm which is $\sim 1$ for the conditions in neutron star crusts. Note that $L$ can be affected slightly by nonquantizing magnetic fields (Yakovlev 1980a).

With $\tau_{\|}^{\kappa, \sigma}=\tau_{0}$, the longitudinal conductivities are equal to those for $B=0: \kappa_{\|}=\kappa_{0}, \sigma_{\|}=1 / \mathcal{R}_{\|}=\sigma_{0}$,

$$
\begin{aligned}
& \kappa_{0} \approx 4.04 \times 10^{15} \frac{x^{3} T_{6}}{\sqrt{1+x^{2}}}\left(\frac{\tau_{0}}{10^{-16} \mathrm{~s}}\right) \frac{\mathrm{erg}}{\mathrm{cm} \mathrm{s} \mathrm{K}}, \\
& \sigma_{0} \approx 1.49 \times 10^{22} \frac{x^{3}}{\sqrt{1+x^{2}}}\left(\frac{\tau_{0}}{10^{-16} \mathrm{~s}}\right) \mathrm{s}^{-1} .
\end{aligned}
$$

Moreover $\mathcal{R}_{\perp}=\sigma_{0}^{-1}$.

It was Lee (1950) who first expressed $\kappa_{0}$ and $\sigma_{0}$ in case (i) through the Coulomb logarithm $L$ and made an estimate of $L$. Later the problem was reconsidered by other authors; see Yakovlev and Urpin (1980) for the references to earlier works. Yakovlev and Urpin (1980) obtained simple analytic expression for $L$ neglecting the electron screening in (10). This screening generally gives a small contribution except at low $\rho$ (near curve $N$ in Fig. 1). Itoh et al. (1983) recalculated $L$ including the electron screening. Yakovlev (1987) showed a noticeable contribution to $L$ (about $40 \%$ for ${ }^{56} \mathrm{Fe}$ ions at $\rho>10^{6} \mathrm{~g} / \mathrm{cm}^{3}$ ) comes from non-Born corrections. However the Born approximation has been used so far in all other calculations.

The scattering on phonons at $B=0$ was also considered in a a number of works (early works are discussed by Yakovlev and Urpin 1980). Raikh and Yakovlev (1982) studied the problem for all $T<T_{m}$ and pointed out that the Umklapp processes (which are most important for high $T$ ) are frozen out at $T \ll T_{u} \sim T_{p} Z^{1 / 3} e^{2} /\left(\hbar v_{F}\right)$. Itoh et al. $(1984,1993)$ reconsidered the problem ignoring the freezing but including the Debye-Waller factor (which was neglected earlier). The authors did not calculate $\kappa_{0}$ and $\sigma_{0}$ directly but used some intermediate results of Raikh and Yakovlev (1982) in an 
approximate manner. Accordingly the results of Itoh et al. $(1984,1993)$ are invalid for $T \ll T_{u}$. It would also be desirable to reconsider the problem for higher $T$.

As follows from (11) the transverse conductivities decrease monotonically with $B$. The Hall conductivities increase linearly with $B$ at small $B$ and decrease at large $B$. The magnetic field strongly affects the transverse conduction when $\eta_{0}>1$. If, in particular, $\eta_{0} \gg 1$ then the transverse conductivities are greatly suppressed $\left(\kappa_{\perp} \approx \kappa_{\|} / \eta_{0}^{2}\right)$ due to rapid Larmor rotation of the electrons. For instance, in the nonrelativistic electron gas at $B \sim 10^{12} \mathrm{G}$ and $T>T_{m}$ the Hall parameter can be as high as $\eta_{0} \sim 10^{2}-10^{3}$. Then the suppression reaches 4 - 6 orders of magnitude.

\subsubsection{Quantizing magnetic fields}

The theory of longitudinal and transverse transport properties of a degenerate electron gas in quantizing magnetic fields is based on the linearized relativistic kinetic equation which describes relaxation of the electron Landau states (Sec. 3) due to electron collisions.

The longitudinal conductivities $\kappa_{\|}$or $\sigma_{\|}$are determined by a set of coupled linear equations for populations of various Landau states in the presence of weak temperature gradients, $\nabla T_{\|}$, or electric fields parallel to $\mathbf{B}$. The electron scattering is assumed to be elastic, and the equations couple the populations of states with the same electron energy. The theoretical approach was developed by Yakovlev (1980a, 1984), who also did the first calculations for all three scattering mechanisms discussed in Sec. 5.1. More extensive calculations were performed by Hernquist (1984), Schaaf (1988) and Van Riper (1988).

Calculations show that in the quantizing magnetic fields $\left(T<T_{B}\right)$ the longitudinal relaxation times $\tau_{\|}^{\kappa, \sigma}$ (as well as the conductivities $\kappa_{\|}, \sigma_{\|}$) display oscillations (Fig. 4) as a function of $\rho$ (or $\mu=\mu(\rho)$ ). The nature of the oscillations is the same as for thermodynamic quantities (Sec. 4). In particular, $\tau_{\|}^{\kappa, \sigma} \ll \tau_{0}$ for $\rho \ll \rho_{B}$ (strongly quantizing field, the ground level is populated only) but $\tau_{\|}$is several times larger than $\tau_{0}$ for $\rho \approx \rho_{B}$. The oscillations are also visible when much higher Landau levels are populated $\left(\rho \gg \rho_{B}\right)$. The oscillations are affected by the thermal broadening of the Landau levels as well as by other broadening mechanisms. Generally, the relaxation times $\tau_{\|}^{\kappa}$ and $\tau_{\|}^{\sigma}$ are different and the Wiedemann-Franz rule does not hold. However one has $\tau_{\|}^{\kappa}=\tau_{\|}^{\sigma}$ in the limit of $T \rightarrow 0$. When the temperature grows and reaches $T_{B}$, the oscillations are smeared out and 


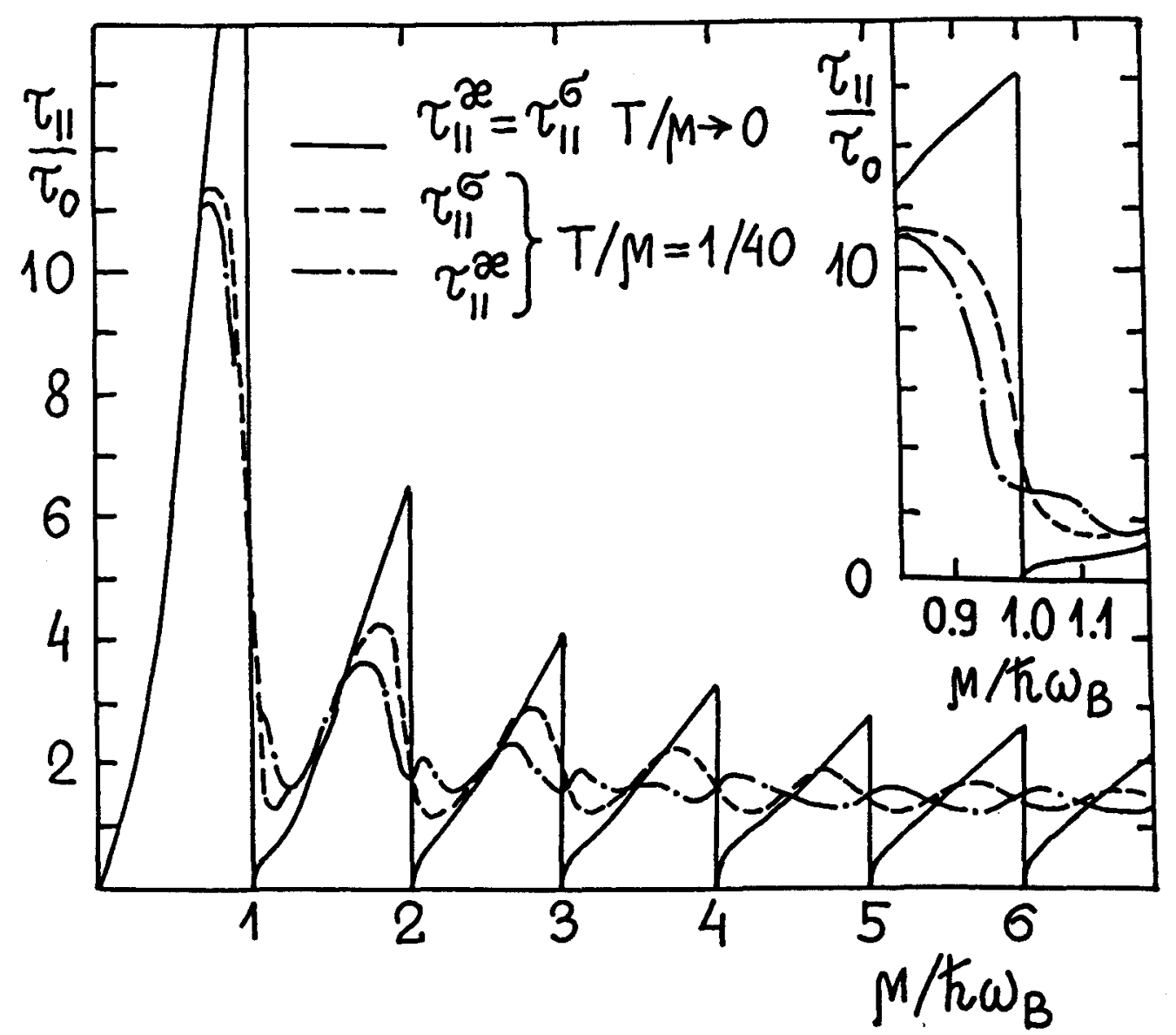

Fig. 10.4 Quantum oscillations of $\tau_{\|}^{\kappa}$ and $\tau_{\|}^{\alpha}$ versus $\mu / \hbar \omega_{B}$ for Coulomb scattering in a nonrelativistic electron gas with ${ }^{56} \mathrm{Fe}$ ions at very strong $\left(k_{B} T / \mu \rightarrow 0\right)$ and finite $\left(k_{B} T / \mu=1 / 40\right)$ degeneracy (Yakovlev 1980a). The curves are self-similar as long as $B<4 \times 10^{13} \mathrm{G} ; \mu$ is the nonrelativistic chemical potential. Population of the Landau levels $n=1-7$ is depicted. Population of the level $n=1$ is shown separately (top right)

the nonquantizing regime is restored, $\tau_{\|}^{\kappa, \sigma}=\tau_{0}$. The oscillations of the longitudinal thermopower are much stronger than those of $\sigma_{\|}$and $\kappa_{\|}$.

The transverse conductivities $\kappa_{\perp}$ and $\sigma_{\perp}$ in quantizing magnetic fields can be presented in the form of closed (but complicated) analytic expressions using the expansion of the general kinetic equation in the inverse Hall parameter, $1 / \eta$, which is small in strong magnetic fields. This theory generalizes the theory of electron transport in terrestrial solids (e.g., Zyrianov and Klinger 1976) to the case of a relativistic electron gas and 


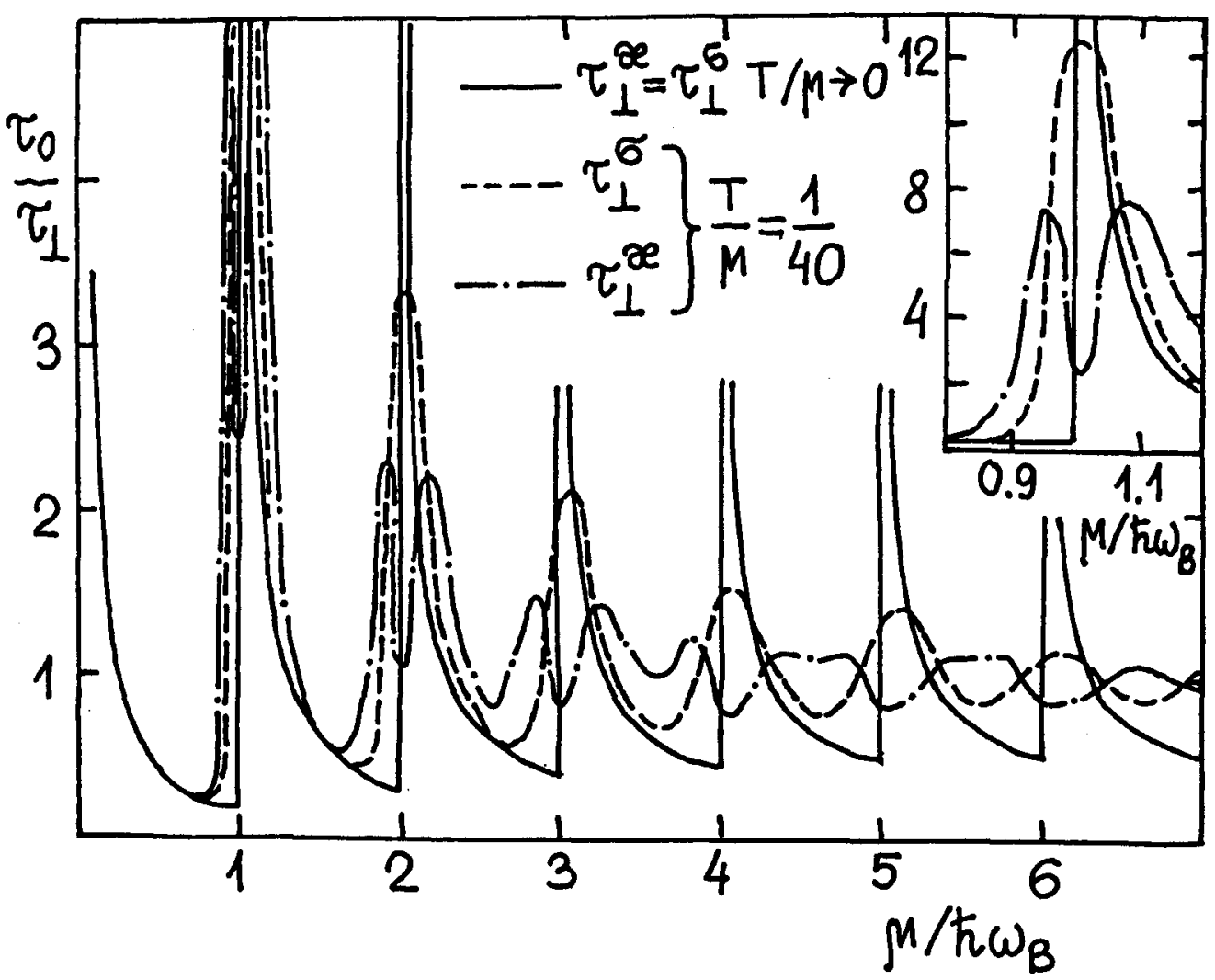

Fig. 10.5 Same as in Fig. 4 for quantum oscillations of $\tau_{\perp}^{\kappa}$ and $\tau_{\perp}^{\sigma}$ (Yakovlev $1980 \mathrm{~b})$. The width of the Landau levels assumed in the calculation is $\gamma=0.1 k_{B} T$

appropriate electron scattering mechanisms. The general expressions for the transverse conductivities in quantizing magnetic fields were obtained by Yakovlev (1980b) and Kaminker and Yakovlev (1981). The expressions include integrals over electron energies which contain logarithmic-type divergencies. The divergencies are removed by introducing the effective widths of the Landau levels (Sec. 4). The widths may be caused by collisional broadening, by some inelasticity of electron scattering and/or by deviations from the Born approximation.

The calculations of the transverse conductivities were carried out by Yakovlev (1980b), and, in more detail, by Hernquist (1984) and Schaaf (1988). The transverse effective relaxation times $\tau_{\perp}^{\boldsymbol{\kappa}}$ and $\tau_{\perp}^{\sigma}$ suffer powerful quantum oscillations (Fig. 5) in the quantizing fields $\left(T<T_{B}\right)$. When the temperature increases to $T_{B}$, the oscillations are completely smeared out 
by the thermal broadenenig of the Landau levels, and the nonquantizing results are reproduced, $\tau_{\|}^{\kappa, \sigma}=\tau_{0}$. Quantum oscillations of $\tau_{\|}^{\kappa, \sigma}$ introduce additional complication into the suppression of $\kappa_{\perp}$ and $\sigma_{\perp}$ by strong magnetic fields. Note that the transverse resistivity $\mathcal{R}_{\perp}$ depends on $B$ only through $\tau_{\perp}^{\sigma}$.

As for the Hall conductivities, $\kappa_{H}$ and $\sigma_{H}$, they suffer no essential quantum oscillations since they are almost independent of the relaxation time at high magnetic fields, see (11).

\subsubsection{Radiative thermal conductivity}

In the very surface layers of neutron stars, where the electrons are nondegenerate or weakly degenerate, heat is transported mainly by photons rather than by electrons. The theoretical expression for the tensor of radiative thermal conductivity in a magnetic field was obtained by Pavlov and Yakovlev (1977). The radiative heat transport is anisotropic. It is determined by two conductivities, $\kappa_{\|}$and $\kappa_{\perp}$, along and across $B$ :

$$
\begin{aligned}
& \kappa_{\|, \perp}=\frac{4}{3} a c T^{3} \overline{l_{\|, \perp}} \\
& \overline{l_{\|, \perp}}=\frac{45}{16 \pi^{4}} \int_{0}^{\infty} d z \frac{z^{4} \mathrm{e}^{z}}{\left(\mathrm{e}^{z}-1\right)^{2}} \int_{0}^{\pi} d \theta \sin \theta l F_{\|, \perp},
\end{aligned}
$$

where $a$ is the Stefan constant, $\overline{l_{\|, \perp}}$ is the generalized Rosseland mean free path of photons along or across $B\left(F_{\|}=2 \cos ^{2} \theta, F_{\perp}=\sin ^{2} \theta\right) ; l=l(\omega, \theta)$ is the mean free path of photons of frequency $\omega=z k_{B} T / \hbar$ averaged over ordinary and extraordinary photon modes which can propagate in a magnetized plasma, and $\theta$ is angle between propagation direction and $\mathbf{B}$.

Pavlov and Yakovlev (1977) and Silant'ev and Yakovlev (1980) calculated $\kappa_{\|, \perp}$ for the cases when the photon mean free path is determined by free-free transitions and/or Thomson scattering. The effect of the magnetic field on the radiative conductivity is described by the parameter $\beta=\hbar \omega_{B} /\left(k_{B} T\right)=$ $T_{B} / T$ (see (8)). If $T \gg T_{B}(\beta \ll 1)$, the field is nonquantizing and its effect is weak, $\kappa_{\|} \approx \kappa_{\perp} \approx \kappa_{0}$. If $T \ll T_{B}(\beta \gg 1)$, the magnetic field is quantizing, and the thermal conductivity is anisotropic and strongly enhanced by the magnetic field, $\kappa_{\|, \perp} \sim \kappa_{0} \beta^{2}$. The enhancement occurs because the main heat carriers at $\beta \gg 1$ are extraordinary mode photons with frequencies $\omega \ll \omega_{B}$. The mean free path of these photons is a factor of $\left(\omega_{B} / \omega\right)^{2}$ larger than at $B=0$. The enhancement $\kappa \propto \beta^{2}$ for $\beta \gg 1$ was first outlined by Tsuruta et al. (1972) and Lodenquai et al. (1974) using a simplified approach. 
An important fact is that $\kappa_{\perp}$ becomes larger than $\kappa_{\|}$in high magnetic fields. For instance, in the case of the Thomson scattering at $\beta \gg 1$ one obtains $\kappa_{\perp} \approx 2 \kappa_{\|} \approx \beta^{2} /\left(2 \pi^{2}\right)$. These asymptotes are valid for large $\beta$ while for intermediate $\beta$ Silant'ev and Yakovlev (1980) got analytic fits

$$
\frac{\kappa_{\|}}{\kappa_{0}}=\frac{\beta^{2}}{4 \pi^{2}}+3 \ln \left(1.40+0.00555 \beta^{2}\right), \frac{\kappa_{\perp}}{\kappa_{0}}=\frac{\beta^{2}}{2 \pi^{2}}+\frac{1+0.0223 \beta^{2}}{1+0.152 \beta^{2}}
$$

where $\kappa_{0}=7.5 \times 10^{20} \mathrm{~T}_{8}^{3} \rho / \mu_{e}$ erg $\mathrm{cm}^{-1} \mathrm{~s}^{-1} \mathrm{~K}^{-1}$, and $\rho$ is expressed in $\mathrm{g}$ $\mathrm{cm}^{-3}$.

For example, consider a helium plasma with $T=4.5 \times 10^{6} \mathrm{~K}$, and $B=2 \times$ $10^{12} \mathrm{G}$. The Thomson scattering dominates at $\rho \ll 1 \mathrm{~g} \mathrm{~cm}^{-3}$. In the latter case $\kappa_{\|} \approx 100 \kappa_{0}$ and $\kappa_{\perp} \approx 180 \kappa_{0}$. If $\rho \gg 1 \mathrm{~g} \mathrm{~cm}^{-3}$, free-free transitions dominate, and the conductivity enhancement is weaker, $\kappa_{\|} \approx 31 \kappa_{0}$ and $\kappa_{\perp} \approx 21 \kappa_{0}$ (now with respect to the free-free conductivity $\kappa_{0}$ for the same $T$ and $\rho$ ). At very low $\rho$ the radiative thermal conductivity is affected by electron - positron vacuum polarization in strong magnetic fields (Pavlov and Yakovlev 1982).

\subsection{Neutrino energy losses in magnetized crusts}

Neutron stars become transparent to neutrinos in half a minute after their birth. Neutrino generation produces powerful energy losses in the cores and crusts of young neutron stars. The main neutrino production mechanisms in the crusts are: electron bremsstrahlung on atomic nuclei $(e+Z \rightarrow e+$ $Z+\nu+\bar{\nu})$, electron-positron annihilation $\left(e^{-}+e^{+} \rightarrow \nu+\bar{\nu}\right)$, plasmon decay $\left(\hbar \omega_{p e} \rightarrow \nu+\bar{\nu}\right)$, and photon decay $(\gamma+e \rightarrow e+\nu+\bar{\nu})$. These processes have been studied in detail for $B=0$ since the classic works of Beaudet et al. (1967) and Festa and Ruderman (1969) (see Itoh et al. 1989 for the references to further works). All processes can be affected by magnetic fields. Moreover magnetic fields lead to a new process, synchrotron radiation of neutrino pairs by electrons $(e \rightarrow e+\nu+\bar{\nu})$, which is forbidden by momentum and energy conservation for $B=0$. Neutrino synchrotron radiation was first studied by Landstreet (1967) but rather qualitatively (see Kaminker et al. 1992a, for critical remarks).

So far two processes have been investigated in strong magnetic fields: the neutrino synchrotron and pair-annihilation radiations. These processes are described by similar matrix elements. According to the Weinberg - Salam theory both processes proceed via charged and neutral currents, and hence all neutrino flavors can be generated. For temperatures $T \ll M_{W} c^{2} \sim 100$ $\mathrm{GeV}$ ( $M_{W}$ being the mass of the intermediate boson), the matrix element 
is described by a 'four-tail' diagram. General (but complicated) expressions for the neutrino energy loss rates $Q_{s}$ and $Q_{p}\left[\mathrm{erg} \mathrm{cm}^{-3} \mathrm{~s}^{-1}\right]$ in synchrotron and pair annihilation processes were obtained by Kaminker et al. (1992a) using a formalism for relativistic electrons in quantizing magnetic fields.

Kaminker et al. (1992a) also calculated $Q_{s}$ and $Q_{p}$ from the general expressions for a nonrelativistic (degenerate and nondegenerate) plasma with strong fields. Kaminker et al. (1991) investigated $Q_{s}$ in a relativistic degenerate electron gas with nonquantizing magnetic fields. Kaminker and Yakovlev (1993) performed a similar investigation for a nondegenerate relativistic electron gas. Kaminker et al. (1992b) examined $Q_{p}$ for a hot nondegenerate relativistic plasma with high magnetic fields. The main results are as follows.

The pair annihilation energy loss $Q_{p}$ is usually unaffected by the magnetic fields in the cases when $Q_{p}$ contributes significantly to the total neutrino energy losses. However the magnetic field does influence $Q_{p}$ if $B \gg 10^{14}$ $\mathrm{G}$ and/or if $B$ is quantizing (Sec. 3). In the latter case the temperature is not too high $\left(T \ll T_{B}\right)$ and annihilation is generally ineffective due to the small number density of positrons.

Synchrotron energy losses $Q_{s}$ can be quite large in a relativistic electron gas (Fig. 6). One should especially mention a wide domain of the relativistic degenerate electron gas with nonquantizing magnetic fields in which $T_{B} \ll$ $T<T_{F}$ and $T<T_{p B}$, where $T_{p B}=1.5 \hbar \omega_{B} x^{2} / k_{B} \approx 2.0 \times 10^{8} B_{12} x^{2} \mathrm{~K}(x$ is given by (1)). According to Kaminker et al. (1991) in this domain

$$
Q_{s}=0.00126 \frac{G^{2} e^{2} B^{2}}{c^{7} \hbar^{8}}\left(k_{B} T\right)^{5} \approx 8.97 \times 10^{14} B_{13}^{2} T_{9}^{5} \frac{\mathrm{erg}}{\mathrm{cm}^{3} \mathrm{~s}},
$$

where $G$ is the Fermi weak interaction constant; $Q_{s}$ includes generation of $\nu_{e}, \nu_{\mu}$ and $\nu_{\tau}$. As follows from (16) $Q_{s}$ depends neither on the electron mass nor on the electron Fermi momentum (i.e. on $\rho$ ). Accordingly all charged relativistic degenerate fermions emit synchrotron neutrino pairs at the same rate, provided the assumed conditions are satisfied. For electrons, this regime occurs in a very wide domain of $\rho, T$ and $B$. A similar regime is possible in the hot relativistic plasma (Kaminker and Yakovlev 1993).

A comparison of the annihilation and synchrotron neutrino energy losses shows that the annihilation process dominates in a hot and not too dense plasma, while the synchrotron process dominates at higher $\rho$ and large $B$. According to the estimates, the synchrotron process may be the most effective neutrino production process in neutron star matter for $T \sim 10^{9} \mathrm{~K}$, $B \geq 10^{13} \mathrm{G}$, and $\rho \sim 10^{7}-10^{8} \mathrm{~g} \mathrm{~cm}^{-3}$. Further work is required for studying other neutrino generation mechanisms in strong magnetic fields. 


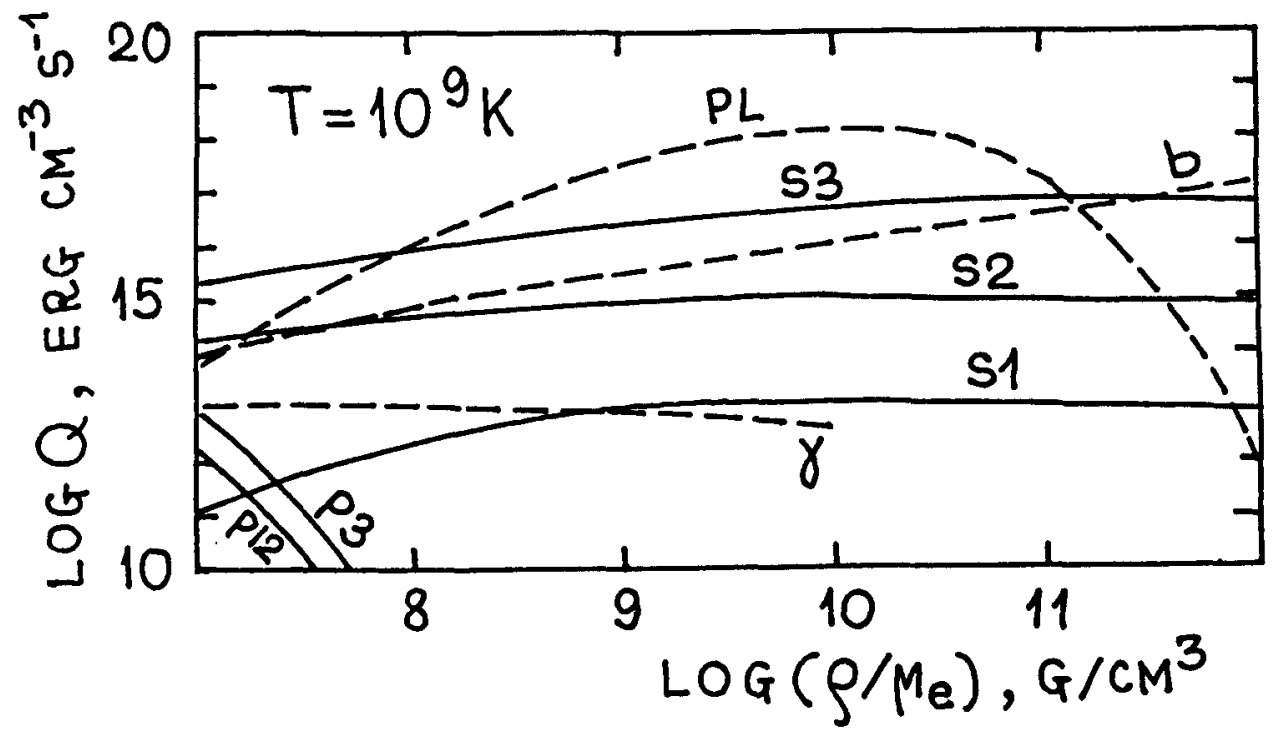

Fig. 10.6 Neutrino energy loss rates versus $\rho / \mu_{e}$ for $T=10^{9} \mathrm{~K}$. Solid lines show synchrotron $(s)$ and pair annihilation $(p)$ losses at $B=10^{12}, 10^{13}$ and $10^{14} \mathrm{G}$ (curves 1,2 , and 3 , respectively). Dashes display bremsstrahlung (b) (Soyeur and Brown 1979, for ${ }^{56} \mathrm{Fe}$ ions), plasmon decay $(p l)$ and photon decay $(\gamma)$ losses (Itoh et al. 1989) for $B=0$. The pair annihilation is much more powerful for higher $T$

\subsection{Structure and thermal evolution of magnetized crusts}

The above results are important for modeling the thermal structure of the surface layers of neutron stars with strong magnetic fields. The modeling is required for investigating cooling and thermal surface radiation (spectra, beaming, polarization) of isolated neutron stars. The cooling and radiation theories are interrelated; they are used for interpretation of observations of thermal radiation from some neutron stars.

Magnetic fields affect most strongly the atmosphere and outermost crust layers of neutron stars. Of particular importance is the layer of the crust across which the bulk of the temperature drop between the interior of the star and the atmosphere occurs. The latter layer is thick (up to several meters) in hot neutron stars whose effective surface temperature $T_{e}$ is higher than some $10^{6} \mathrm{~K}$. However the layer is much thinner for cooler stars. The magnetic field becomes important provided it affects this layer. In the latter case the temperature distribution is not spherically symmetric within the isolating layer due to anisotropic heat transport. The atmosphere acts as a transmitter: it creates spectral, angular and polarization features of the 
outgoing radiation which passes through the atmosphere from the crust. In particular, the radiation spectrum may deviate noticeably from the blackbody one (Romani 1987).

The strongest effects of the magnetic fields occur in the atmosphere. Although we do not consider atmospheres in this paper we note that huge magnetic fields distort the structure of atoms, ions and molecules in atmospheric layers (see, e.g., Lai et al. 1992, Pavlov and Mészáros 1993, and references therein). The distortion takes place particularly by electric fields induced in the center-of-mass frames of particles moving across $B$. The magnetic fields influence the ionization equilibrium, equation of state, other thermodynamic properties of matter, and the radiative spectral opacities. Radiation transfer in magnetized atmospheres (Shibanov et al. 1992) differs significantly from that in non-magnetic atmospheres.

Deeper in the crust, magnetic field effects become weaker.

The full self-consistent theory of magnetized atmospheres and deeper surface layers of neutron stars has not yet been developed. It should take into account the effects of magnetic fields on the equation of state and anisotropic thermal conductivities of crust layers. It is most likely that the thermal conductivity is unable to keep the heat balance, and additional slow flows of matter of the meridional circulation type (e.g., Schwarzschild 1958) are generated. The circulation may drag the magnetic field, distort its configuration and induce magnetic forces sufficiently large that they are important in the balance of forces. The solution of these problems should yield the temperature variation at the bottom of the atmosphere, which provides the necessary boundary conditions for the radiative transfer problem in the atmosphere.

So far the above problems have been solved in a simplified manner. Magnetic fields have been considered as force-free, although their effects on the equation of state (Sec. 4) have been included. Possible circulation flows and magnetic forces have been neglected.

The density profiles in cold $(T=0)$ neutron star crusts with magnetic fields have been calculated recently by Rögnvaldsson et al. (1993) on the basis of the TF equation of state (Sec. 4, Fig. 2). The equation of hydrostatic equilibrium has been integrated for constant magnetic field in the plane-parallel approximation. The binding of matter introduced by strong magnetic fields $\left(\rho<\rho_{B}\right)$ leads to higher surface densities. In deep layers, $\rho \gg \rho_{B}$, the density profiles tend to those for $B=0$.

The temperature profiles in magnetized crusts were analyzed by Hernquist (1985), using the plane-parallel approximation. Later the most extensive studies of temperature profiles in a crust with constant vertical magnetic 


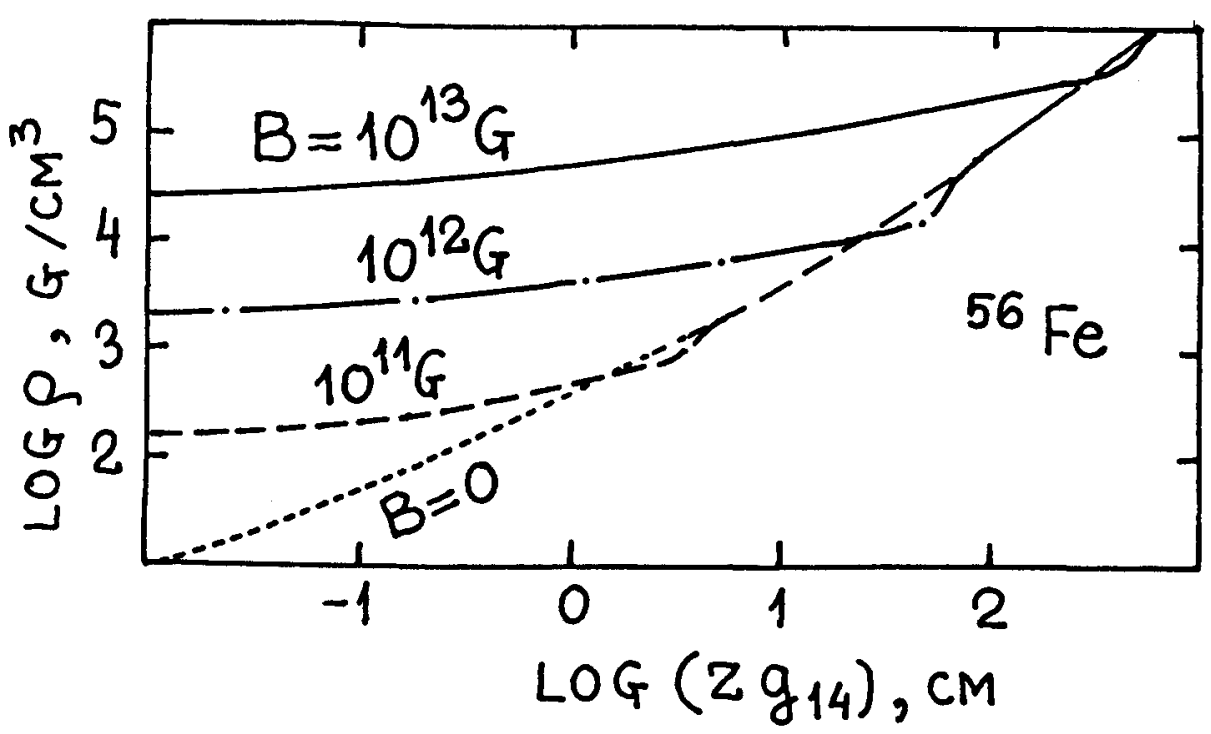

Fig. 10.7 Density profiles versus depth $z$ (measured from the surface) in a cold $(T=0)$ neutron star crust with magnetic field (Rögnvaldsson et al. 1993); $g_{14}$ is surface gravity in units of $10^{14} \mathrm{~cm} \mathrm{~s}^{-2}$

field were performed by Van Riper (1988), again in the plane-parallel approach. In this case heat is transported by the longitudinal (electron and radiative) conductivity $\kappa_{\|}$. The effects of the magnetic fields on the equation of state were included. The relation between the internal temperature $T_{i}$ and the effective surface temperature $T_{e}$ obtained by Van Riper (1988) is shown in Fig. 8. In hot crusts $\left(T_{e}>10^{6} \mathrm{~K}\right)$, the isolating surface layer is rather thick $\left(\rho \leq 10^{6} \mathrm{~g} \mathrm{~cm}^{-3}\right.$ ) and the relation is slightly affected by the magnetic fields. Accordingly the $T_{e}\left(T_{i}\right)$ dependence appears to be close to the field-free one (Urpin and Yakovlev 1979, Gudmundsson et al. 1983, Hernquist and Applegate 1984). For cooler stars $\left(T_{e}<10^{6} \mathrm{~K}\right)$, the isolating layer shifts to lower densities. It becomes thinner and field dependent. For a given $T_{i}, T_{e}$ grows with $B$. The growth occurs because the thermal isolation is weaker when $B$ is larger. First, the low-density matter is 'removed' by the magnetic binding (Fig. 7 ); second the radiative thermal conductivity is enhanced (Sec. 5.4) making the isolation poorer.

A two-dimensional analysis of thermal conduction in the surface layers of a neutron star has been performed by Schaaf (1990). The magnetic field has been assumed constant both in magnitude and direction in the layers under study. The anisotropic thermal conductivity has been taken into account. Many simplifications introduced in the calculation make the results rather 


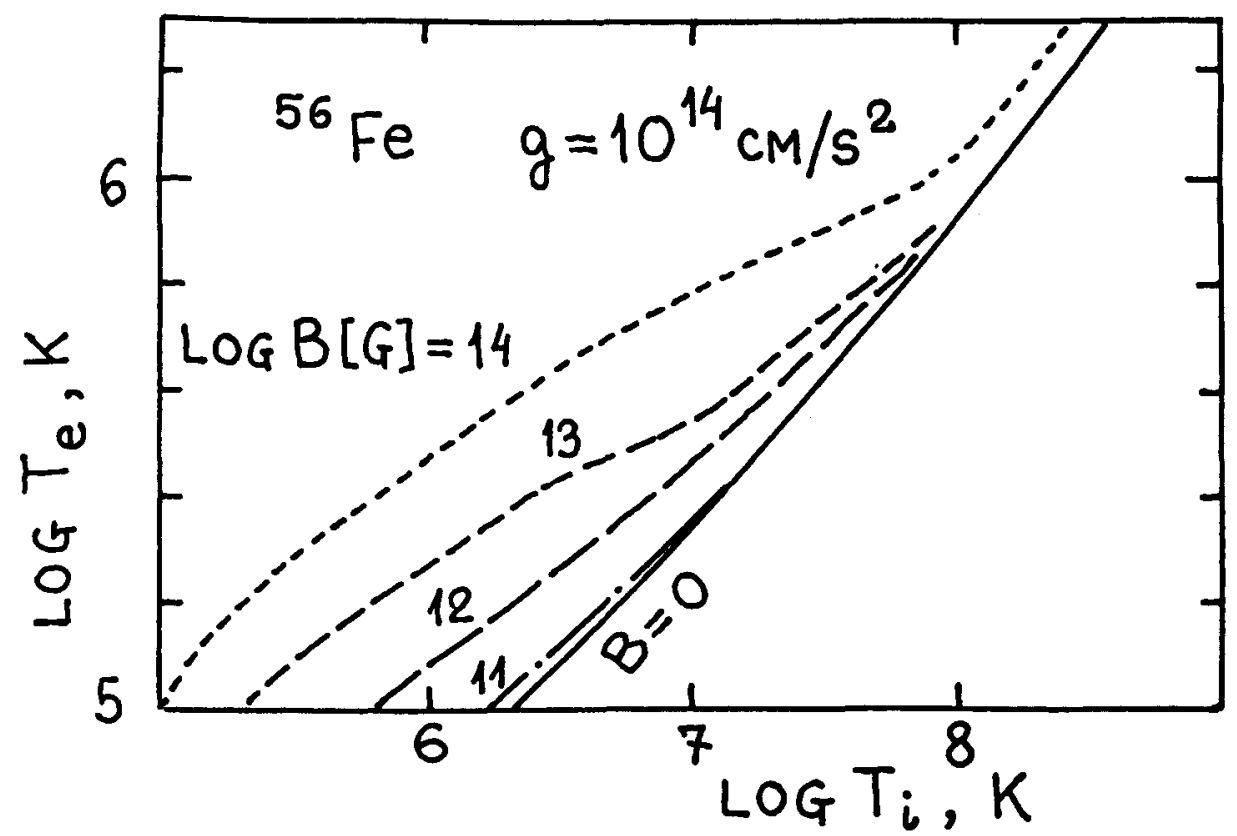

Fig. 10.8 Effective surface temperature $T_{e}$ (not redshifted) versus internal temperature $T_{i}$ for a neutron star with surface gravity $g=10^{14} \mathrm{~cm} \mathrm{~s}^{-2}$ at several values of $B$ (from Fig. 29 of Van Riper 1988)

indecisive. However the main conclusions seem to be qualitatively correct. The magnetic field affects the surface temperature distribution if the star is sufficiently cool (as discussed above). The surface temperature at the magnetic equator appears to be lower than at the pole. This is mainly because the thermal isolation of the stellar interior is stronger at the equator. the heat propagates across $B$ in the equatorial layers, and the transverse thermal conductivity of degenerate electrons is strongly suppressed by high fields (Sec. 5). For instance, according to Schaaf (1990) the surface temperature at the equator of the star with $B=10^{11} \mathrm{G}$ (and $g=10^{14} \mathrm{~cm} \mathrm{~s}^{-2}$ ) is about half of that at the pole provided the pole temperature is about $4 \times 10^{5} \mathrm{~K}$. Currently the problem is being reconsidered by Van Riper and Miralles (private communication).

The magnetic fields in the neutron star crust influence cooling (Fig. 9), especially when the star is sufficiently cold $\left(T_{e}<10^{6} \mathrm{~K}\right)$. Detailed cooling calculations of magnetized neutron stars have been carried out by Van Riper (1991) using the $T_{e}\left(T_{i}\right)$ relationship obtained by Van Riper (1988). The magnetic field leads to higher surface temperatures $T_{e}(t)$ during the neutrino cooling stage $\left(t<10^{5}-10^{6} \mathrm{yrs}\right)$ when the neutrino luminosity is larger than the photon surface luminosity. At this stage, the internal stellar 


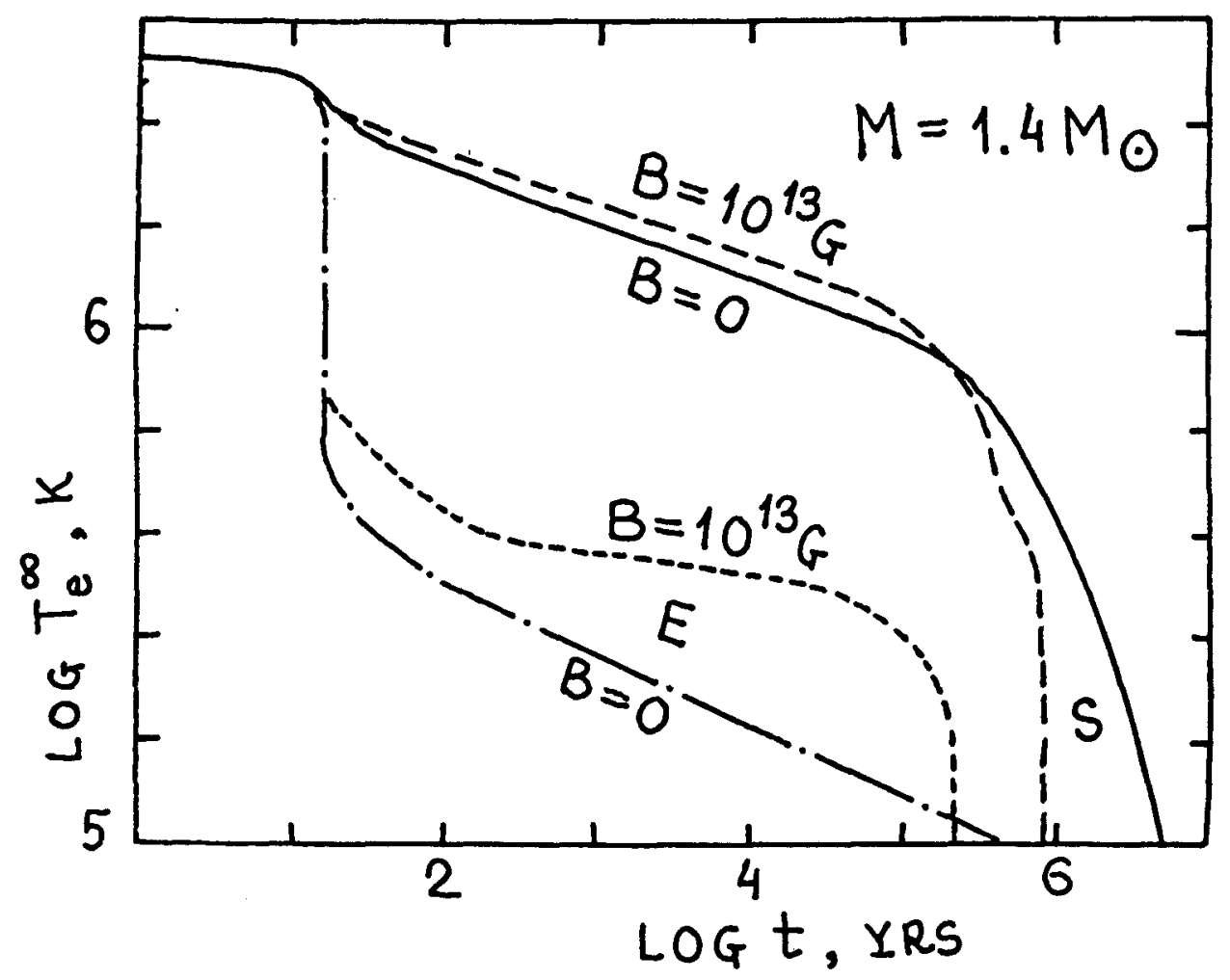

Fig. 10.9 Decrease of the effective surface temperature (redshifted) with time for a neutron star (Friedman - Pandharipande equation of state in dense core) with standard neutrino luminosity $(s)$ or with the luminosity enhanced $(e)$ by the presence of quark matter in stellar interior (from Fig. 10 of Van Riper 1991)

temperature is controlled by the neutrino emission and does not depend on the surface magnetic field. Accordingly $T_{e}$ is larger if $B$ is stronger. Later the star cools via photon surface emission. The magnetic field reduces thermal isolation of the stellar interior. This accelerates photon cooling and leads to a lower $T_{e}(t)$ (Fig. 9). If some enhanced neutrino production mechanism, such as neutrino emission by free quarks or the direct URCA process in a hyperonic core, operates in the stellar interior (see Pethick 1993 and references therein), the effect of the field on cooling is naturally more pronounced. 


\subsection{Conclusions}

Recent results of the ROSAT X-ray observatory (Finley et al. 1992, Becker et al. 1993, Anderson et al. 1993) indicate that surface thermal radiation has most likely been detected from at least five neutron stars: PSR 0656+14, PSR 1929+10, PSR 1055-52, Geminga, and the Vela pulsar. All these objects are known to possess large magnetic fields. The detected radiation contains information on neutron star masses, radii, magnetic fields, as well on dense matter in stellar interiors: on the equation of state, and the absence or presence of enhanced cooling mechanisms, etc. So far theoretical interpretations of the observations are rather uncertain. The effective surface temperatures of the neutron stars appear to be model dependent, but they do not deviate significantly from those predicted by standard cooling theories (without enhanced neutrino luminosity). The exception is PSR $0656+14\left(t \approx 10^{5} \mathrm{yrs}\right)$ whose surface temperature seems to be lower than the standard one but higher than that predicted by the fully allowed enhanced cooling $\left(T_{e}=(4-8) \times 10^{5} \mathrm{~K}\right.$, for most realistic theoretical fits of the radiation spectra detected, e.g. Anderson et al. 1993). In this case, one possibility is that cooling is enhanced by the direct URCA process in the stellar core but the enhancement is partly suppressed by nucleon superfluidity (e.g., Page and Applegate 1992). More precise observations and more elaborate theories are required to arrive at definite conclusions.

Future theoretical studies of neutron star crusts with strong magnetic fields should be based on the theoretical results described above. However there are still many theoretical problems to be solved. The most important of them are concerned with the effects of magnetic fields in the atmospheres, and the surface layers of neutron crusts as discussed in Sec. 7.

The above results are also important for analyzing generation and evolution of magnetic fields in neutron stars. These problems are not discussed in the present work (see, e.g., Blandford et al. 1983, Urpin et al. 1986, Urpin and Van Riper 1993, and the references therein).

We are grateful to Yu.A. Shibanov and K. Van Riper for discussions and comments. We are also extremely thankful to C. Pethick for careful reading of the manuscript and many important critical remarks. This work was supported partly by Russian Foundation of Fundamental Researches, grant No. 93-02-2916.

\section{References}

Anderson S.B., Córdova F.A., Pavlov G.G., Robinson C.R., and Thompson R.J., Ap. J. 414, 867 (1993) 
Beaudet G., Petrosian V. and Salpeter E.E., Ap. J. 150, 979 (1967)

Baym G., Pethick C.J. and Sutherland P., Ap. J. 170, 299 (1971)

Becker W., Trümper J. and Ögelman H., in: Isolated Pulsars, K. Van Riper, R.

Epstein, C. Ho eds., Cambridge: Cambridge Univ. Press, 104 (1993)

Blandford R.D. and Hernquist L., J. Phys. C 15, 6233 (1982)

Blandford R.D., Applegate J.H. and Hernquist L., MNRAS 204, 1025 (1983)

Canuto V. and Ventura J., Fundam. Cosmic Phys. 2, 203 (1977)

Carr W.J., Phys. Rev. 122, 1437 (1961)

Ceperley D.M. and Alder B.J., Phys. Rev. Lett. 45, 566 (1980)

Festa G.G. and Ruderman M.A., Phys. Rev. 180, 1227 (1969)

Finley, J.P., Ögelman, H., Kiziloğlu, Ü., Ap. J. 394, L21 (1992)

Gudmundsson E.H., Pethick C.J. and Epstein R.I., Ap. J. 272, 286 (1983)

Hansen J.-P., Phys. Rev. A8, 3096 (1973)

Hansen J.-P., Torrie G.M. and Vieillefosse P., Phys. Rev. A16, 2153 (1977)

Hernquist L., Ap. J. Suppl. 56, 325 (1984)

Hernquist L., MNRAS 213, 313 (1985)

Hernquist L. and Applegate J.H., Ap. J. 287, 224 (1984)

Itoh N., Mitake S., Iyetomi H., and Ichimaru S., Ap. J. 273, 774 (1983)

Itoh N., Kohyama Y., Matsumoto N., and Seki M., Ap. J. 285, 758 (1984); erratum 404, 418

Itoh N., Adachi T., Nakagawa M., Kohyama Y., and Munakata H., Ap. J. 339, 354 (1989)

Itoh N., Hayashi H. and Kohyama Y., Ap. J. submitted (1993)

Kaminker A.D. and Yakovlev D.G., Teor. Mat. Fiz. 49, 248 (1981)

Kaminker A.D. and Yakovlev D.G., Zh. Eksper. Teor. Fiz. 103, 283 (1993)

Kaminker A.D., Levenfish K.P. and Yakovlev D.G., Pisma Astron. Zh. 17, 1090 (1991)

Kaminker A.D., Levenfish K.P., Yakovlev D.G., Amsterdamski P., and Haensel P., Phys. Rev. D46, 3256 (1992a)

Kaminker A.D., Gnedin O.Yu., Yakovlev D.G., Haensel P., and Amsterdamski P., Phys. Rev. D46, 4133 (1992b)

Klepikov N.P., Zh. Eksper. Teor. Fiz. 26, 19 (1954)

Kubo R., Miyake S. and Hashitsume N., Solid State Phys. 17, 269 (1965)

Lai D., Salpeter E. and Shapiro S.L., Phys. Rev. A45, 4832 (1992)

Landau L.D. and Lifshitz E.M., Statistical Physics, Part I, Pergamon: Oxford (1980)

Landstreet J.D., Phys. Rev. 153, 1372 (1967)

Lee T.D., Ap. J. 111, 625 (1950)

Lodenquai J., Canuto V., Ruderman M. and Tsuruta S., Ap. J. 190, 141 (1974)

Lorenz C.P., Ravenhall D.G. and Pethick C.J., Phys. Rev. Lett. 70, 379 (1993)

O'Connell R.F. and Roussel K.M., Astron. Astrophys. 18, 198 (1972)

Mochkovitch R. and Hansen J.-P., Phys. Lett. A73, 35 (1979)

Nagara H., Nagata Y. and Nakamura T., Phys. Rev. A36, 1859 (1987)

Negele J.W. and Vautherin D., Nucl. Phys. A207, 298 (1973)

Page D. and Applegate J.H., Ap. J. 394, L17 (1992)

Pavlov D.G. and Mészáros P., Ap. J. submitted (1993)

Pavlov D.G. and Yakovlev D.G., Astrofizika 13, 173 (1977)

Pavlov D.G. and Yakovlev D.G., Astrofizika 18, 119 (1982)

Pethick C.J., Rev. Mod. Phys. 64, 1133 (1992) 
Pines D., in: Neutron Stars: Theory and Observation, J. Ventura and D. Pines eds., p. 57, Kluwer Acad. Publ.: Dordrecht (1991)

Pollock E.L. and Hansen J.-P., Phys. Rev. A8, 3110 (1973)

Raikh M.E. and Yakovlev D.G., Astrophys. Space Sci. 87, 193 (1982)

Rögnvaldsson Ö.E., Fushiki I., Gudmundsson E.H., Pethick C.J., and Yngvason J., Preprint No. 93/9A Nordita, Copenhagen (1993); Ap. J., in press

Romani R., Ap. J. 331, 718 (1987)

Schaaf M.E., Astron. Astrophys. 205, 335 (1988)

Schaaf M.E., Astron. Astrophys. 235, 499 (1990)

Schmid-Burgk J., Astron. Astrophys. 26, 335 (1973)

Schoenberg D., Magnetic Oscillations in Metals Cambridge Univ. Press: Cambridge (1984)

Schwarzschild M., Structure and Evolution of the Stars, Princeton University Press: Princeton (1958)

Shapiro S.L. and Teukolsky S.A., Black Holes, White Dwarfs, and Neutron Stars, Wiley - Interscience: New York (1983)

Shibanov Yu.A., Zavlin V.E., Pavlov G.G., and Ventura J., Astron. Astrophys. 266, 313 (1992)

Silant'ev N.A. and Yakovlev D.G., Astrophys. Space Sci. 71, 45 (1980)

Slattery W.L., Doolen G.D. and DeWitt H.E., Phys. Rev. A21, 2087 (1980)

Slattery W.L., Doolen G.D. and DeWitt H.E., Phys. Rev. A26, 2255 (1982)

Soyeur M. and Brown G.E., Nucl. Phys. A324, 464 (1979)

Tsuruta S., Canuto V., Lodenquai J., and Ruderman M., Ap. J. 176, 739 (1972)

Urpin V.A. and Van Riper K., Ap. J. 411, L87 (1993)

Urpin V.A. and Yakovlev D.G., Astrofizika 15, 647 (1979)

Urpin V.A. and Yakovlev D.G., Sov. Astron. 24, 425 (1980)

Urpin V.A., Levshakov S.A. and Yakovlev D.G., MNRAS 219, 703 (1986)

Usov N.A., Grebenschchikov Yu.B. and Ulinich F.R., Zh. Eksper. Teor. Fiz. 78, 296 (1980)

Van Riper K., Ap. J. 329, 339 (1988)

Van Riper K., Ap. J. Suppl. 75, 449 (1991)

Wambach J., Ainsworth T.L. and Pines D., in: Neutron Stars: Theory and Observation, J. Ventura and D. Pines eds., p. 37, Kluwer Acad. Publ.: Dordrecht (1991)

Yakovlev D.G., Preprint No. 678, Ioffe Phys. Techn. Inst., Leningrad (1980a)

Yakovlev D.G., Preprint No. 679, Ioffe Phys. Techn. Inst., Leningrad (1980b)

Yakovlev D.G., Astrophys. Space Sci. 98, 37 (1984)

Yakovlev D.G., Astron. Zh. 64, 661 (1987)

Yakovlev D.G. and Shalybkov D.A., Sov. Sci. Rev. / Sec. E, R.A. Syunyaev ed., 7, 311 (1989)

Yakovlev D.G. and Urpin V.A., Sov. Astron. 24, 303 (1980)

Zyrianov P.S. and Klinger M.I., Quantum Theory of Electron Transport Phenomena in Crystalline Semiconductors , Nauka: Moscow (1976) 